\title{
Risco à saúde representado pelo consumo de leite cru comercializado clandestinamente*
}

\section{Health risk due to the consumption of raw milk commercialized without due authorization}

\author{
Kathia Brienza Badini, Antonio Nader Filho, Luiz Augusto do Amaral e Pedro Manuel Leal \\ Germano \\ Faculdade de Medicina Veterinária da Universidade de Marília. Marília, SP - Brasil (K.B.B.); \\ Departamento de Medicina Veterinária Preventiva da Faculdade de Ciências Agrárias e Veterinárias \\ de Jaboticabal da Universidade Estadual Paulista. Jaboticabal, SP - Brasil (A.N.F., L.A.A.); \\ Departamento de Prática de Saúde Pública da Faculdade de Saúde Pública da Universidade de São \\ Paulo. São Paulo, SP - Brasil (P.M.L.G.)
}

\begin{abstract}
Resumo
Foram submetidas às contagens de microrganismos mesófilos, Staphylococccus coagulase positiva, e às determinações do número mais provável (NMP) de coliformes totais e de coliformes fecais, 60 amostras de leite cru comercializado clandestinamente nos Municípios de Botucatu e de São Manuel, Estado de São Paulo, Brasil. Os resultados obtidos evidenciaram a ocorrência de $41(68,3 \%)$ e $50(83,3 \%)$ amostras com contagens de microrganismos mesófilos e de coliformes totais, respectivamente, acima dos limites máximos estabelecidos pelo Ministério da Saúde para o leite pasteurizado tipo C. Evidenciaram, ainda, a presença de $30(50,0 \%)$ e $11(18,3 \%)$ amostras contaminadas por cepas de Staphylococcus coagulase positiva e por coliformes fecais, respectivamente. Apenas $5(8,3 \%)$ amostras mostraram-se dentro dos referidos padrões legais.
\end{abstract}

Leite, microbiologia. Enterobacteriaceae, isolamento \& purificação. Staphylococcus, isolamento \& purificação.

\begin{abstract}
Sixty raw milk samples commercialized without due authorization in the counties of Botucatu and S. Manuel, State of S. Paulo (Brazil), were submitted to mesophilic microorganism and coagulase-positive Staphylococcus and most probable number of total coliform and fecal coliform counts. Forty-one $(68.3 \%)$ and $50(83.3 \%)$ of the samples were found, respectively to contain mesophilic microorganisms and total coliforms above the maximum limits established by the Health Ministry for type C pasteurized milk. Thirty (50.0\%) and $11(18.3 \%)$ of the samples were found, respectively, to the contaminated by coagulase-positive Staphylococcus and fecal coliforms. Only 5 (8.3\%) samples
\end{abstract}

\footnotetext{
* Parte da dissertação de mestrado apresentada ao Curso de Pós-Graduação em Medicina Veterinária Preventiva da Faculdade de Ciências Agrárias e Veterinárias de Jaboticabal/UNESP, em 1995.

Correspondência para/Correspondece to: Antonio Nader Filho - Departamento de Medicina Veterinária Preventiva da Faculdade de Ciências Agrárias e Veterinárias de Jaboticabal da Universidade Estadual Paulista. Campus de Jaboticabal - Rodovia Carlos Tonanni Km 5 - 14870-000 Jaboticabal, SP - Brasil.

Edição subvencionada pela FAPESP. Processo 95/2290-6.

Recebido em 19.10.1995. Aprovado em 29.5.1996.
} 
were found to comply with the required legal standards. The results showed the unsatisfactory hygienic and sanitary conditions of the raw milk and suggest the existence of great risk to the health of the consumers, especially when the product is taken without being boiled.

Milk microbiology. Enterobacteriaceae, isolation. Staphylococcus, isolation.

\section{INTRODUÇÃO}

Apesar da proibição legal imposta à comercialização do leite cru no Brasil (Lei n ${ }^{\circ} 1.283$ de 18/12/ 1950 e Decreto ${ }^{\circ} 30.691$ de 29/03/1952), a venda deste tipo de leite tem sido realizada abertamente na periferia da cidade de São Paulo, assim como em numerosas cidades do Estado, muitas delas possuidoras de elevado nível socioeconômico e cultural (Queiroz ${ }^{8}$, 1995). Situação semelhante pode ser observada em Minas Gerais, onde estima-se que $47 \%$ do leite consumido pela população sejam oriundos da comercialização clandestina do leite cru (Brandão $\left.{ }^{1}, 1994\right)$.

Tais afirmações são preocupantes principalmente se considerado o fato de o leite cru poder veicular inúmeros microrganismos patogênicos ao homem (Robinson' ${ }^{9}$ 1987). A este respeito a Organização Mundial de Saúde comprovou a existência de sete enfermidades viróticas básicas e de 16 bacterianas veiculadas por este produto (Brandão ${ }^{1}$, 1994).

Considerando a destacada importância que o leite assume na alimentação humana, realizou-se o presente trabalho com o objetivo de conhecer as características microbiológicas do leite cru comercializado clandestinamente nos Municípios de Botucatu/SP e de São Manuel/SP, de modo a obter subsídios que permitam avaliar o risco potencial que este produto pode representar para a saúde da população consumidora.

\section{MATERIAL E MÉTODO}

Foram identificados 10 produtores rurais que comercializavam clandestinamente o leite cru no Município de Botucatu/SP e 10 no Município de São Manuel/SP. Em seguida, procedeu-se à identificação de um consumidor do produto comercializado para cada produtor rural, em ambos os municípios.

Foram mantidos contatos com os consumidores, e esclarecidos os objetivos do trabalho, solicitando-lhes colaboração para fornecerem as amostras de leite que seriam objeto das análises microbiológicas.

Optou-se pela colheita das amostras de leite cru nos domicílios dos consumidores. Caso este procedimento fosse realizado diretamente nas propriedades rurais perder- se-ia a oportunidade de verificar as prováveis influências do binômio tempo/temperatura sobre as características microbiológicas do produto, fato este que impossibilitaria a avaliação da qualidade deste tipo de leite, no momento da sua distribuição ao consumo.

A grande maioria dos consumidores que concordaram em colaborar para a realização desta investigação recebia o produto em sua residência, o qual era transportado em veículos de tração animal e automotivos. No dia anterior ao da colheita de amostras, eram entregues nos domicílios dos referidos consumidores frascos de vidro com tampa e capacidade para $1.000 \mathrm{ml}$, previamente esterilizados. Desse modo, no momento da entrega, com o auxílio da "caneca" utilizada pelo produtor durante a comercialização, o consumidor procedia o transvase do produto para os referidos recipientes.

Foram colhidas 3 amostras em cada domicílio, durante 3 semanas consecutivas, de modo a totalizar 60 amostras procedentes dos 20 produtores rurais de ambos os municípios estudados. Após o acondicionamento em caixas de material isotérmico ("isopor"), contendo cubos de gelo, as amostras eram transportadas para os laboratórios do Departamento de Higiene Veterinária e Saúde Pública da Faculdade de Medicina Veterinária e Zootecnia da UNESP, "Campus" de Botucatu, onde eram realizadas as análises microbiológicas.

As contagens de microrganismos mesófilos e de Staphylococcus coagulase positiva, assim como a determinação do número mais provável (NMP) de coliformes totais e de coliformes fecais, foram realizadas pelas técnicas recomendadas pelo Ministério da Agricultura ${ }^{3}$ (1981).

Adotou-se como referencial para a análise e interpretação dos resultados, os padrões microbiológicos estabelecidos pelo Ministério da Saúde ${ }^{4}$ (1987) para o leite pasteurizado tipo $\mathrm{C}$, pois além de constituir-se no produto de maior consumo em nosso meio, os padrões exigidos para este tipo de leite apresentam menor rigor que os estabelecidos para os dos tipos A e B.

\section{RESULTADOS E DISCUSSÃO}

Na Tabela 1 verifica-se que dentre as 60 amostras de leite cru comercializado clandestinamente nos Municípios de Botucatu/SP e de São Manuel/SP, 41 $(68,3 \%)$ apresentaram contagens de microrganismos mesófilos acima de $300.000 \mathrm{UFC} / \mathrm{ml}$, valor este estabelecido como sendo o limite máximo permitido pelos padrões do Ministério da Saúde, para o leite pasteurizado tipo C. Observa-se, ainda, que a média 
Tabela 1 - Distribuição das contagens de microrganismos mesófilos em amostras de leite cru comercializado clandestinamente nos Municípios de Botucatu/ SP e de São Manuel/SP, 1993.

\begin{tabular}{lccc}
\hline \multirow{2}{*}{$\begin{array}{c}\text { Microrganismos } \\
\text { mesófilos } \\
(\text { UFC/ml x 1.000) }\end{array}$} & \multicolumn{2}{c}{ Amostras de leite cru } \\
\cline { 2 - 4 } 1 & 300 & 19 & $\mathrm{n}^{\circ}$ \\
300 & 1.000 & $4^{*}$ & 31,7 \\
1.000 & 5.000 & $9^{*}$ & 6,7 \\
5.000 & 10.000 & $2^{*}$ & 3,0 \\
10.000 ou mais & $26^{*}$ & 43,3 \\
\hline \multicolumn{2}{c}{ Total } & 60 & 100,0 \\
\hline Média aritmética: 97.498.700 UFC/ml & \\
* Amostras fora dos padrões legais estabelecidos pelo Ministério da Saúde para \\
o leite pasteurizado tipo C
\end{tabular}

aritmética das referidas contagens foi de 97.498 .700 $\mathrm{UFC} / \mathrm{ml}$.

Queiroz $^{8}$ (1995) obteve resultados muito semelhantes ao analisar o leite cru comercializado clandestinamente nos municípios de Juquitiba/SP e de Itapecerica da Serra/SP, onde constatou a ocorrência de $38(63,3 \%)$ amostras de leite cujas contagens de microrganismos mesófilos situaram-se acima do referido valor, sendo que a média aritmética destas contagens foi de $95.634 .900 \mathrm{UFC} / \mathrm{ml}$.

Tais achados eram esperados, pois além de não sofrer qualquer tipo de tratamento térmico, este produto era mantido à temperatura ambiente por um largo período de tempo, desde a sua obtenção até a chegada ao domicílio do consumidor.

A Tabela 2 mostra a ocorrência de $50(83,3 \%)$ amostras nas quais o NMP de coliformes totais situou-se acima de $5 / \mathrm{ml}$, valor este estabelecido como sendo o limite máximo permitido pelos padrões do Ministério da Saúde para o leite pasteurizado tipo C. Este achado é muito semelhante ao obtido por Queiroz $^{8}$ (1995) que verificou a ocorrência de 49 $(81,7 \%)$ amostras nas quais o NMP de coliformes

Tabela 2 - Distribuição do número mais provável (NMP) de coliformes totais e de coliformes fecais em amostras de leite cru comercializado clandestinamente nos Municípios de Botucatu/SP e de São Manuel/SP, 1993.

\begin{tabular}{|c|c|c|c|c|c|}
\hline \multirow{2}{*}{\multicolumn{2}{|c|}{$\begin{array}{c}\text { Número } \\
\text { mais provável } \\
(\mathrm{NMP} / \mathrm{ml})\end{array}$}} & \multicolumn{2}{|c|}{ Coliformes totais } & \multicolumn{2}{|c|}{ Coliformes fecais } \\
\hline & & $\mathrm{n}^{\circ}$ & $\%$ & $\mathrm{n}^{\circ}$ & $\%$ \\
\hline \multicolumn{2}{|c|}{ Zero } & 10 & 16,7 & 49 & 81,7 \\
\hline $10^{1}$ & $10^{3}$ & $3 *$ & 5,0 & $1 *$ & 1,6 \\
\hline $10^{3}$ & $10^{4}$ & $18^{*}$ & 30,0 & $3 *$ & 5,0 \\
\hline $10^{4}$ & $10^{6}$ & $15^{*}$ & 25,0 & $4^{*}$ & 6,7 \\
\hline \multicolumn{2}{|c|}{$10^{6}$ ou mais } & $14^{*}$ & 23,3 & $3 *$ & 5,0 \\
\hline \multicolumn{2}{|c|}{ Total } & 60 & 100,0 & 60 & 100,0 \\
\hline
\end{tabular}

* Amostras fora dos padrões legais estabelecidos pelo Ministério da Saúde para o leite pasteurizado tipo C
Tabela 3 - Distribuição das contagens de Staphylococcus coagulase positiva em amostras de leite cru comercializado clandestinamente nos Municípios de Botucatu/SP e de São Manuel/SP, 1993.

\begin{tabular}{|c|c|c|c|}
\hline \multirow{2}{*}{\multicolumn{2}{|c|}{$\begin{array}{l}\text { Staphylococcus } \\
\text { coagulase positiva } \\
(\mathrm{UFC} / \mathrm{ml} \times 1.000)\end{array}$}} & \multicolumn{2}{|c|}{ Amostras de leite cru } \\
\hline & & $\mathrm{n}^{\circ}$ & $\%$ \\
\hline \multicolumn{2}{|c|}{ Zero } & 30 & 50,0 \\
\hline 1 & 1.000 & $6 *$ & 10,0 \\
\hline 1.000 & 5.000 & $10^{*}$ & 16,7 \\
\hline 5.000 & 10.000 & $4^{*}$ & 6,7 \\
\hline 10.000 & 100.000 & $9 *$ & 15,0 \\
\hline \multicolumn{2}{|c|}{100.000 ou mais } & $1 *$ & 1,6 \\
\hline \multicolumn{2}{|c|}{ Total } & 60 & 100,0 \\
\hline
\end{tabular}

totais superou o limite máximo permitido pelos referidos padrões legais. A exemplo do que ocorreu com as contagens de microrganismos mesófilos, tais achados também eram esperados em função dos mesmos motivos acima referidos.

Os dados da Tabela 2 revelam, ainda, a ocorrência de $11(18,3 \%)$ amostras contaminadas por bactérias pertencentes ao grupo dos coliformes fecais. Este valor é muito inferior ao obtido por Queiroz ${ }^{8}$ (1995) que observou a ocorrência de $39(65,0 \%)$ amostras contaminadas pelos referidos microrganismos. Tais diferenças talvez possam ser atribuídas às características peculiares do processo de obtenção do leite nos distintos municípios, objeto dessas investigações.

A Tabela 3 mostra a ocorrência de $30(50,0 \%)$ amostras contaminadas por Staphylococcus coagulase positiva, portanto, em desacordo com os padrões estabelecidos pelo Ministério da Saúde, que determina a ausência destes microrganismos em amostras de leite pasteurizado tipo C. Verifica-se, ainda, que a média aritmética das contagens dos referidos microrganismos foi de $9.885 \mathrm{UFC} / \mathrm{ml}$. Queiroz ${ }^{8}$ (1995) obteve resultados inferiores, uma vez que

Tabela 4 - Distribuição do número de análises microbiológicas fora dos padrões legais, segundo as amostras de leite cru comercializado clandestinamente nos Municípios de Botucatu/SP e de São Manuel/SP, 1993.

\begin{tabular}{ccc}
\hline $\begin{array}{c}N^{\circ} \text { de análises micro- } \\
\text { biológicas fora dos } \\
\text { padrões legais* }\end{array}$ & \multicolumn{2}{c}{ Amostras de leite cru } \\
\cline { 2 - 3 } & $\mathrm{n}^{\circ}$ & $\%$ \\
\hline Zero & 5 & 8,3 \\
Uma & 10 & 16,7 \\
Duas & 24 & 40,0 \\
Três & 12 & 20,0 \\
Quatro & 9 & 15,0 \\
\hline Total & 60 & 100,0 \\
\hline
\end{tabular}

* Estabelecidos pelo Ministério da Saúde para o leite pasteurizado tipo C 
constatou a ocorrência de $24(40,0 \%)$ amostras contaminadas pelo Staphylococcus coagulase positiva, cujas contagens apresentaram uma média aritmética de $3.996 \mathrm{UFC} / \mathrm{ml}$.

Os dados inseridos na Tabela 4 revelam que apenas 5 amostras de leite cru $(8,3 \%)$ apresentaram-se com características microbiológicas dentro dos padrões legais estabelecidos pelo Ministério da Saúde para o leite pasteurizado tipo C. Tais achados evidenciam a péssima qualidade microbiológica deste tipo de leite, que se mostrou inferior à do leite pasteurizado tipo $\mathrm{C}$, conforme evidenciam as investigações realizadas por Silveira e col. ${ }^{10}$, 1988; Nader Filho e col. ${ }^{5}$, 6,7 , 1988, 1989, 1990 e Cerqueira e col. ${ }^{2}, 1994$. Esses citados autores, ao analisarem as características microbiológicas do leite pasteurizado tipo C, comercializado nas cidades de São Paulo/SP, Barretos/ SP, Jaboticabal/SP, Ribeirão Preto/SP e de Belo Hori-

\section{REFERÊNCIAS BIBLIOGRÁFICAS}

1. BRANDÃO, S.C.C. Leite: legislação, reponsabilidade e saúde pública. Rev. Balde Branco, 360: 68-71, 1994.

2. CERQUEIRA, M.M.D.P.; SOUZA, M.R.; RODRIGUES, R.; FONSECA, C.M.; RUBINICK, J.; QUINTAES, I.A.S. Características microbiológicas do leite cru e beneficiado em Belo Horizonte. Arq. Bras. Med. Vet. Zoot., 46: 713-22, 1994.

3. MINISTÉRIO DA AGRICULTURA. Métodos analíticos oficiais para controle de produtos de origem animal e seus ingredientes. I- Métodos Microbiológicos. Brasília, 1981.

4. MINISTÉRIO DA SAÚDE. Secretaria Nacional de Vigilância Sanitária. Portaria no 1 de 28/1/87. Padrões Microbiológicos para Alimentos. Diário Oficial da União de 12/2/87, Seção I. p. 2.197-2.199.

5. NADER FILHO, A.; ROSSI JÚNIOR, O.D.; SCHOCKENITURRINO, R.P. Características microbiológicas do leite pasteurizado tipo C, comercializado em Barretos, São Paulo. Ars Vet., 4: 291-6, 1988.

6. NADER FILHO, A.; ROSSI JÚNIOR, O.D.; SCHOCKENITURRINO, R.P. Avaliação das características
zonte/MG, verificaram que $73,6 \%, 44,5 \%, 68,4 \%$, $86,4 \%$ e $80,0 \%$ das amostras, respectivamente, apresentavam-se dentro dos citados padrões legais.

Considerando-se a inexistência de qualquer tipo de fiscalização e/ou controle higiênico-sanitário dos animais que estão envolvidos com a produção deste tipo de leite, assim como do processo de obtenção, transporte e comercialização, tais achados constituem-se em motivo de preocupação, principalmente em função do risco representado pelo consumo deste produto sem que tenha sido previamente submetido à fervura.

\section{AGRADECIMENTO}

Ao Prof.Dr.Aristides Cunha Rudge (FMVZ/ UNESP), pelo valioso apoio oferecido quando da realização das análises microbiológicas. microbiológicas do leite pasteurizado tipo $\mathrm{C}$ e das embalagens plásticas utilizadas no envase, em uma usina de beneficiamento do Estado de São Paulo, Brasil. Rev. Microbiol., 20: 261- 6,1989.

7. NADER FILHO, A.; AMARAL, L.A.; ROSSI JÚNIOR, O.D.; LUIZ, A.F. Características microbiológicas do leite pasteurizado dos tipos A, B e C, comercializados na cidade de Ribeirão Preto, Estado de São Paulo. Ars Vet., 6: 166-72, 1990.

8. QUEIROZ, J.C. Avaliação sanitária do leite cru distribuído nos Municípios de Juquitiba e Itapecerica da Serra, São Paulo, 1990-1992, 1995. [Tese de Doutorado - Faculdade de Saúde Pública da USP].

9. ROBINSON, R.K. Microbiologia lactológica. Zaragoza, Acribia, 1987. v. 1

10. SILVEIRA, N.V.V.; SAKIMA, H.; DUARTE,M.; RODAS, M.A.B.; CHICOUREL,E.L. Avaliação das condições físicoquímicas e microbiológicas do leite pasteurizado consumido na cidade de São Paulo. Rev. Inst. Lact. Cândido Tostes, 260: $40-5,1988$. 